\title{
Double Photoionization of Endohedrally Confined Atoms
}

\author{
F. D. Colavecchia, ${ }^{1}$ G. Gasaneo, ${ }^{2}$ and D. Mitnik $^{2}$ \\ ${ }^{1}$ División Colisiones Atómicas, Centro Atómico Bariloche and Conicet, Río Negro 8400 S. C. de Bariloche, Argentina \\ ${ }^{2}$ Departamento de Física, Instituto de Astronomía y Física del Espacio, \\ Universidad de Buenos Aires and CONICET, 1428 Buenos Aires, Argentina
}

Correspondence should be addressed to F. D. Colavecchia, flavioc@cab.cnea.gov.ar

Received 29 September 2010; Accepted 7 December 2010

Academic Editor: Roberto Daniel Rivarola

Copyright $\odot 2011$ F. D. Colavecchia et al. This is an open access article distributed under the Creative Commons Attribution License, which permits unrestricted use, distribution, and reproduction in any medium, provided the original work is properly cited.

\begin{abstract}
We study the double electronic emission by photon impact from ground state of two-electron atoms in the center of a model spherical fullerene, which is described by a square-well shell. Cross-sections for different well depth are computed within a separable model for the final state, and a configuration interaction state for the initial one. Triple differential cross-sections show a strong dependence on the well depth and on the energy of the emitted electrons, due to the delocalization of the electrons in the initial state. The fullerene potential also allows higher angular momenta partial waves to be included in the process, which modifies the well-known two-lobe cross-section from isolated atom.
\end{abstract}

\section{Introduction}

Fullerene molecules, formed by pentagonal and hexagonal arrangements of atoms, have received attention right from its discovery and undoubtedly opened many new captivating areas in physics. These molecules come in different sizes and shapes, from the well-known, quasispherical $\mathrm{C}_{60}$ carbon fullerene [1] to nanotubes. Beyond the specific features of fullerenes, many methods to include atoms inside their shells have been developed [2, 3]. These compounds form stable molecules that also attracted the interest of the community, such as their potential use as nanocages to store atoms $[4,5]$. Many properties of these systems are determined by the differences in the physics of the embedded atom compared to the isolated one.

Some of the most interesting of these features arise when these endohedrally embedded atoms interact with light and electronic emission occurs during the process. Since the pioneering work of Puska and Nieminen [6], different kinds of resonant behavior have been identified. First evidence of these effects were observed in the photoionization of $\mathrm{C}_{60}$, revealed as oscillations in the cross-sections [7] and attributed to the ability of the carbon shell to support an intramolecular standing wave. Confinement resonances are predicted for endohedrally embedded atoms such as
Xe@ $\mathrm{C}_{60}[8,9]$, due to the reflection of the photoelectron in the fullerene cage. Furthermore, the role of multielectronic correlation has been investigated recently, giving rise to the so-called correlation confinement resonances [10] and also the interference among resonances [11]. However, all these works involved a complex multielectronic atom inside the fullerene shell, that gives rise to many mechanisms of electronic emissions even when the atom is isolated.

Few works are devoted to multiple photoionization of fullerenes or endohedrally embedded atoms. Double-tosingle total cross-sections ratio has been measured recently for isolated $\mathrm{C}_{60}$, which exhibits interesting modulation properties [12]. Kidun et al. have examined the multiple photoionization of $\mathrm{C}_{60}$ within a many-particle approach [13]. Two-electron photoionization has been studied for high photon energies by Amusia et al. [14], but only the doubleto-single photoionization cross-section ratio was reported. They analyzed the process with highly asymmetrical energy sharing, where the energy of one electron is much bigger than the energy of the other one. The emission in that case proceeds with a shake-off mechanism, where the fast electron absorbs most of the energy of the photon, while the slow one is ejected due to the residual modified atomic field left after the ejection of the first one. Since their model of the fullerene potential was a delta function, it did not 
take into account accurately the possible delocalization of the atomic electrons into the cage. Recently, Pindzola et al. analyzed the double ionization of $\mathrm{He}_{0} \mathrm{C}_{60}$, and found that there is only a change in magnitude in the triply differential cross-sections, whereas there are some differences in single and total cross-sections due to the confinement resonances [15].

Whereas the investigation of bound or continuum states of two-electron systems can be performed by different techniques, double photoionization (DPI) of atomic or molecular species by single photons has unique advantages. First, it allows to probe the dynamics of the electron pair both in the initial and final states with the same collisional process. Besides, it is free of long-range correlations between the target and the incoming particle. Finally, the complete absorption of the photon by both electrons is determined by the interelectronic correlation. Then, it is not surprising that this process has been thoroughly studied along the years for both atomic and molecular species [16].

\section{The Model for DPI}

In this work we bring a different perspective to the problem of DPI from endohedrally embedded atoms. We choose the simplest system to study the role of a model fullerene cage in the electronic emission as well as the influence of the interelectronic correlation in the process, both in the bound state as well as in the continuum one. Moreover, we do not aim to solve the complete problem consisting on hundreds of electrons. On the contrary, our approach uses a very simple model, both for the atomic wavefunctions and the fullerene potential. To this end, we consider a He-like atom in the center of the fullerene cage and assume that there are only two active electrons in the system. We also focus on equal energy sharing conditions for the ejected photoelectrons. The simplified electronic structure of the fullerene molecule seen by an electron has been usually described through a cage model potential [6]:

$$
V_{w}(r)= \begin{cases}-U_{0} & \text { if } r_{c} \leq r \leq r_{c}+\Delta \\ 0, & \text { otherwise. }\end{cases}
$$

For $\mathrm{C}_{60}, r_{c}=5.75$ a.u., $\Delta=1.89$ a.u. [6, 7]. These simple assumptions enable one to discover all the richness of these systems. Moreover, different spherical fullerenes are described varying the well depth. In fact, the energetic structure of these molecules as a function of the magnitude of the cage potential $U_{0}$ presents a manifold of avoided crossing between states, even within this two-electron model [17, 18]. Similar results were found for endohedrally embedded hydrogen [19]. For the sake of simplicity, we choose to analyze the crossing between the ground and first excited states. Thus, the initial state of the system will be written in terms of one-electron 1s $\left(\phi_{1 s}(r)=4 \sqrt{2} \exp (-2 r)\right)$ and $2 s\left(\phi_{2 s}(r)=2 \exp (-r)(1-r)\right)$ states of the isolated atom, and the approximate solution of the model potential (1), $\phi_{w}(r)=\exp \left(-\alpha\left(r-r_{0}\right)^{2}\right)$ where $r_{0}=r_{c}+\Delta / 2$. We construct the following configuration interaction (CI) wave function with these one-electron wave functions for the bound state:

$$
\begin{aligned}
\Psi_{i}\left(\mathbf{r}_{1}, \mathbf{r}_{2}\right)=N\{ & a \varphi_{1 s}\left(r_{1}\right) \varphi_{1 s}\left(r_{2}\right) \varphi_{\text {corr }}\left(r_{12}\right) \\
& +b\left[\varphi_{1 s}\left(r_{1}\right) \phi_{w}\left(r_{2}\right)+1 \longleftrightarrow 2\right] \\
& \left.+c\left[\varphi_{1 s}\left(r_{1}\right) \varphi_{2 s}\left(r_{2}\right)+1 \longleftrightarrow 2\right] \varphi_{\text {corr }}\left(r_{12}\right)\right\},
\end{aligned}
$$

where $\varphi_{\text {corr }}\left(r_{12}\right)=1+r_{12} / 2$ is a correlation factor.

This state is suitable to analyze the collision process near the crossing between the ground and the first excited state of the system as a function of the potential depth. The basis parameters $a, b, c$ as well as the exponential factor $\alpha$, the normalization constant $N$ and the energy depend on $U_{0}$ and are obtained with variational techniques, see Table 1. The crossing is at $U_{0}^{\text {cross }}=1.35$ a.u. (This value for the crossing depends on the model used for the initial state.) We also choose two other values of the magnitude of the cage potential $U_{0}$ to calculate the cross-sections, one below ( $U_{0}=1.1$ a.u.) and one above $\left(U_{0}=1.65\right.$ a.u. $)$ the crossing of the levels. This particular selection of the basis does enhance the interplay between the free atomic ground state and the partially delocalized state with one electron in the atom and the other one in the fullerene, since the contribution of the $1 s 2 s$ state through coefficient $c$ is much smaller than the other ones. Thus, the basis parameters measure the degree of localization of the electrons: when $a \approx 1$, both electrons are near the atomic core, while $b \approx 1$ implies that the electronic density spreads up to the fullerene cage. The ground state thus changes along the crossing from an almost pure $1 s^{2}$ configuration ( $a \approx 1, b \approx 0)$ to a delocalized state $(a \approx 0$, $b \approx 1$ ) for $U_{0}=1.65$ a.u., while the excited state "mirrors" this behavior.

There are some indications that nondipolar (quadrupolar) terms are important in the single photoionization of endohedrally confined atoms [20], mostly for photon energies near ionization threshold. Besides, endohedral confinement has been found to modify the quadrupolar resonances of the Xe atoms [21]. However, recent calculations show that nondipolar effects in DPI of He are important only for high photon energies [22]. Since experimental data is obtained usually in collisions with low-energy photons [16] we will assume a dipolar approximation for the DPI operator.

The triply differential cross-section (TDCS) in terms of the momenta $\mathbf{k}_{1}$ and $\mathbf{k}_{2}$ of the two ejected electrons can be obtained in the velocity gauge in terms of the transition matrix

$$
T^{(V)}\left(\mathbf{k}_{1}, \mathbf{k}_{2}\right)=\left\langle\Psi_{f}^{-}\left(\mathbf{r}_{1}, \mathbf{r}_{2}\right)\left|\mathcal{E} \cdot\left(\nabla_{1}+\nabla_{2}\right)\right| \Psi_{i}\left(\mathbf{r}_{1}, \mathbf{r}_{2}\right)\right\rangle,
$$

where $\varepsilon$ is polarization of the incoming light. We recall that this calculation can be performed also in acceleration or length gauges. Each gauge form emphasizes different regions of the configuration space, but all of them should give the same theoretical description of the process, provided that both the initial $\Psi_{i}\left(\mathbf{r}_{1}, \mathbf{r}_{2}\right)$ and final $\Psi_{f}^{-}\left(\mathbf{r}_{1}, \mathbf{r}_{2}\right)$ states are exact wave functions, or at least very good approximations for them. Otherwise, differences between gauges are 
TABLE 1: Energies and wave functions parameters (in atomic units) for ground and first excited states of an He-like atom embedded in the model fullerene cage.

\begin{tabular}{ccccccc}
\hline$U_{0}$ & $E$ & $N\left(\times 10^{-2}\right)$ & $\alpha$ & $a$ & $b$ & $c$ \\
\hline \multicolumn{7}{c}{ Ground State } \\
\hline 1.10 & -2.8892 & 4.9390 & 0.47419 & -0.99835 & 0.00187 & 0.05723 \\
1.35 & -2.9002 & 1.54985 & 0.54823 & -0.94505 & -0.31958 & 0.06879 \\
1.60 & -3.0905 & 0.54473 & 0.61216 & -0.06843 & -0.99762 & -0.00798 \\
\hline \multicolumn{6}{c}{ First Excited State } \\
\hline 1.10 & -2.7127 & 0.55145 & 0.47419 & 0.38490 & -0.91623 & 0.11117 \\
1.35 & -2.8879 & 4.71427 & 0.54823 & 0.99770 & -0.03930 & -0.05509 \\
1.60 & -2.8890 & 4.94203 & 0.61216 & 0.99837 & -0.00609 & -0.05671 \\
\hline
\end{tabular}

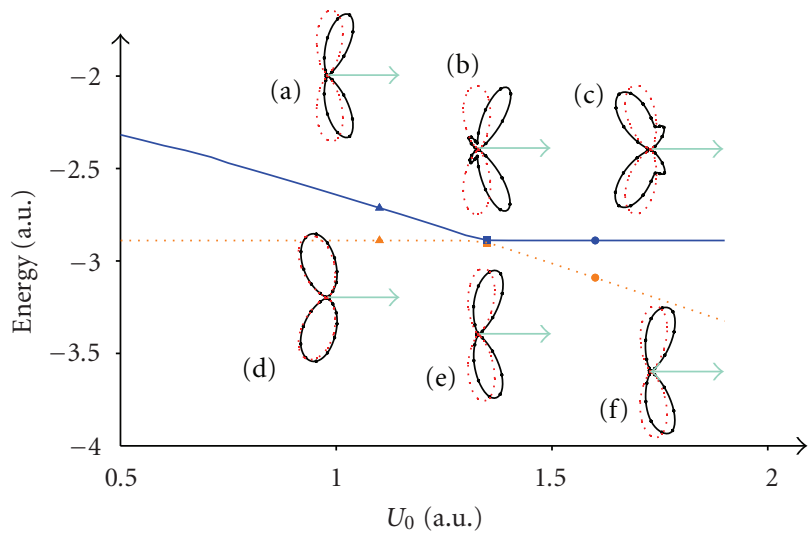

Figure 1: (Color online) Threefold differential cross-section (TDCS) for $(\gamma, 2 e)$ ionization of the helium ground state in a fullerene cage, as a function of the angle of one of the ejected electrons $\theta_{2}$. The other one is ejected at $\theta_{1}=0^{\circ}$, fixed respect to the polarization $\varepsilon$. The polarization vector is set along the $x$-axis, and the impinging light towards the page. The electrons are ejected with equal energy $E_{1}=E_{2}=10 \mathrm{eV}$. Dotted (orange) and solid (blue) lines represent the ground and excited state energy as a function of $U_{0}$, respectively. TDCS are shown for both excited $(\mathrm{a}, \mathrm{b}, \mathrm{c})$ and ground states $(\mathrm{d}, \mathrm{e}, \mathrm{f})$ and computed with a pure Coulomb final state, dashed (red) line or with exact potential including fullerene cage, solid (black) line. They are shown for $U_{0}=1.1$ a.u. (triangles), $U_{0}=1.35$ a.u. (squares) and $U_{0}=1.6$ a.u. (circles). All TDCS are rescaled to one at maxima.

apparent $[23,24]$. For simple systems such as He, these differences are restricted to the magnitude of the crosssections, and minor deviations in the angular distributions [25] that are irrelevant for the mainly qualitative study presented here. The calculation of the transition matrix is performed by direct six-dimensional numerical integration in the electronic spherical coordinates $\mathbf{r}_{1}$ and $\mathbf{r}_{2}$, using a nondeterministic Vegas algorithm, with a relative error is smaller than 3\% in the TDCS for all energies and angles considered [26].

Let us turn our attention to the final state of the ionized electrons in the continuum. Among many approximate wave functions for this state, we choose a simple C3-style wave function (see [27] and references therein):

$$
\Psi_{C 3}^{\text {cage }}\left(\mathbf{r}_{1}, \mathbf{r}_{2}\right)=\psi_{\mathbf{k}_{1}}^{-}\left(\mathbf{r}_{1}\right) \psi_{\mathbf{k}_{2}}^{-}\left(\mathbf{r}_{2}\right) D\left(\alpha_{12}, \mathbf{k}_{12}, \mathbf{r}_{12}\right),
$$

where the electronic wave function in the combined field of the atomic core $(-2 / r)$ and the fullerene cage $\left(V_{w},(1)\right)$ is described by the two-body functions $\psi_{\mathbf{k}_{i}}^{-}\left(\mathbf{r}_{i}\right)$ expanded in partial waves. For comparison purposes, we also make use of a pure Coulomb wave $\psi_{C 3}^{\mathrm{Coul}}\left(\mathbf{r}_{1}, \mathbf{r}_{2}\right)$, where the role of the cage potential is neglected. The electron-electron correlation is modeled with the usual Coulomb distortion factor $D\left(\alpha_{12}, \mathbf{k}_{12}, \mathbf{r}_{12}\right)={ }_{1} F_{1}\left(\alpha_{12}, 1, i \mathbf{k}_{12} \mathbf{r}_{12}+i k_{12} r_{12}\right)$ in terms of the Sommerfeld parameter $\alpha_{12}=1 / k_{12}$ and the relative momentum $\mathbf{k}_{12}=\mathbf{k}_{1}-\mathbf{k}_{2}$. This model correctly reproduces the asymptotic condition of the problem. We recall that, although this model would not lead to accurate absolute differential cross-sections, it accounts for all the relevant features of the collisional process for this system.

\section{Results}

We computed emission in an equal energy sharing situation $\left(E_{1}=E_{2}=10 \mathrm{eV}\right)$, with linearly polarized light. The calculations of TDCS as a function of the magnitude of the fullerene cage $U_{0}$ are displayed in Figure 1. Overall, the cross-sections interchange their character as the states go through the avoided crossing, due to the mirror collapse of the initial state [17]. This is more evident for final Coulomb states (compare red lines in Figure 1(a) with Figure 1(f); or Figure 1(d) with Figure 1(c)).

The emission from the ground state below the crossing (Figure 1(d)) fully agrees with the isolated atom DPI from a $1 s^{2}$ state as expected, and is similar for both final state used, $\psi_{C 3}^{\text {Coul }}\left(\mathbf{r}_{1}, \mathbf{r}_{2}\right)$ or $\psi_{C 3}^{\text {cage }}\left(\mathbf{r}_{1}, \mathbf{r}_{2}\right)$. Near and beyond the avoided crossing, the presence of the fullerene cage counteracts the interelectronic repulsion, moving the lobes towards the emission direction of electron 1 (Figure 1(d) to Figure 1(f)). This is slightly more important in the calculation that includes the cage in the final state (black curves in Figure 1 online).

However, the excited state exhibits a more dramatic change along the crossing: the cross-section changes from the typical two-lobe configuration to a four-lobe one. Thus, the mirror collapse observed in the intial state wave functions is broken in the cross-sections. This effect can be clearly seen in Figures 1(a)-1(c) when compared with Figures $1(\mathrm{~d})-1(\mathrm{f})$, and it is present for both final states used, although it is noticeable at this energy only with the nonpure Coulomb final state $\psi_{C 3}^{\text {cage }}\left(\mathbf{r}_{1}, \mathbf{r}_{2}\right)$. Both the initial and final states contribute to this feature. On one hand, the presence of a delocalized electron far from the nucleus in the initial state slightly changes the interelectronic repulsion. This has already been observed in calculations of double photoionization from $\mathrm{He}(1 \mathrm{~s} 2 \mathrm{~s})$ states [28] and were also attributed to the increasing extension of the initial state. Also, similar effects from the initial state have been measured from $\mathrm{Ne}\left(2 s^{2}\right)[29,30]$. On the other hand, the introduction of the cage potential in the final state $\psi_{C 3}^{\text {cage }}\left(\mathbf{r}_{1}, \mathbf{r}_{2}\right)$ plays a very 


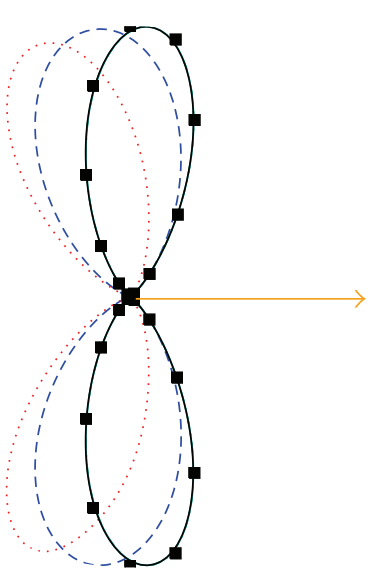

(a)

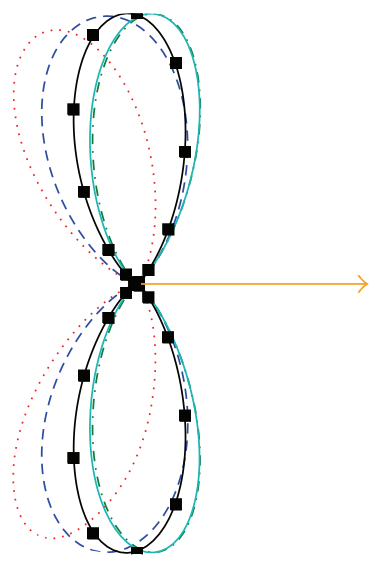

(b)

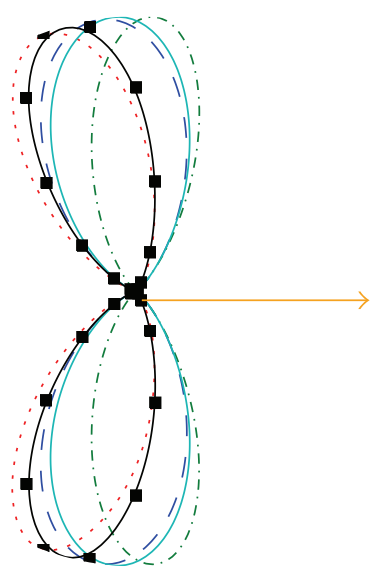

(c)

Figure 2: (Color Online) Contributions of the each initial CI states to the TDCS for $E_{1}=E_{2}=20 \mathrm{eV}$, calculated with the pure Coulomb final state for the DPI of the excited state of endohedrally embedded He. Kinematics as well as values of $U_{0}$ are the same as in Figures $1(\mathrm{a})-1(\mathrm{c})$. Solid (black online) line and squares: full TDCS, solid (cyan online) line, coherent contribution; dashed (blue) line, TDCS from first term, (2); dash-dotted (green) line, TDCS from second term, (2); dotted (red) line, TDCS from third term, (2). All TDCS are rescaled to one at maxima.

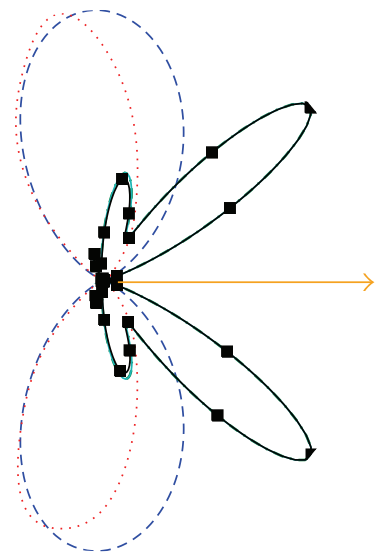

(a)

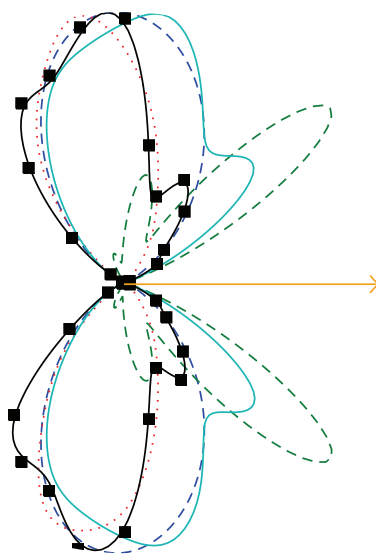

(b)

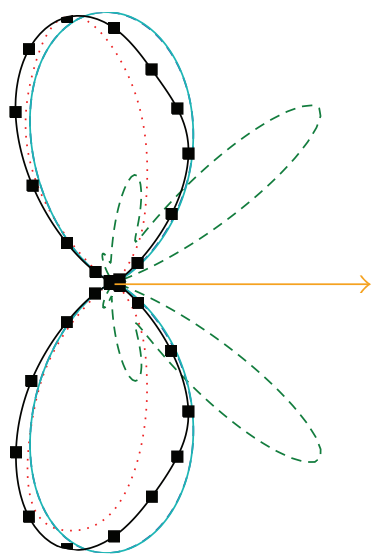

(c)

FIgure 3: (Color Online) Same as Figure 2, with $E_{1}=E_{2}=50 \mathrm{eV}$.

important role, restraining the interelectronic repulsion (Figure 1(b)) or enhancing it (Figure 1(c)) for different well depths.

It is clear that the emission from the excited state deserves further investigation. The simple CI that we adopted to describe the initial state enables us to isolate the role of each $\mathrm{CI}$ state in the cross-sections. We computed the contribution of each CI state separately for the DPI of the excited state, see Figure 2. In this case, the energy sharing is $E_{1}=E_{2}=$ $20 \mathrm{eV}$, and we choose a pure Coulomb wave $\psi_{\mathrm{C} 3}^{\mathrm{Coul}}\left(\mathbf{r}_{1}, \mathbf{r}_{2}\right)$ for the final state. Contribution of the CI atomic states $\left(1 s^{2}\right.$ or $1 \mathrm{~s} 2 \mathrm{~s}$ of (2)) results in the typical emission of electron 2 perpendicular to the direction of electron 1 (dashed and dotted lines in Figure 2). However, the contribution of the atom-well CI state presents a complete different structure, with three clearly distinguishable lobes. It is clear from this figure that the overall shape of the emission is mainly dictated by the interplay between CI states, and not by the simple superposition of them. Below the energy crossing, the crosssection is dominated by the atom-well state (Figure 2(a)), while above it resembles the typical two-lobe emission from $1 s^{2}$ double photoionization (Figure 2(c)). In these cases, the contribution of the coherent sum determines the crosssections, whereas the role of the interference among states clearly defines the behavior at the crossing, see Figure 2(b).

We can now compare the behavior of TDCS for different emission energies. While in Figure 1(b) $\left(E_{1}=E_{2}=10 \mathrm{eV}\right)$ two lobes are well defined when a pure Coulomb final wave is used, six lobes can be observed in Figure 3(b) for $E_{1}=E_{2}=$ $50 \mathrm{eV}$. This is not surprising, since the electronic density of the atom-well CI state has a maximum centered at the cage. Besides, partial waves with higher single electron angular momentum contribute to the cross-section for increasing electron energies and are included in the process, which 
results in these structures in the cross-sections. This is an effect due to the presence of the cage, because for isolated atoms, the centrifugal barrier inhibits the penetration of high angular momenta partial waves into the region where the atomic electronic density is significant. We have checked that this effect is enhanced when the final state $\Psi_{C 3}^{\text {cage }}\left(\mathbf{r}_{1}, \mathbf{r}_{2}\right)$ that includes the fullerene cage is used. Using the expansion proposed by Malegat et al. [31, 32] for the cross-sections, we obtained that the maximum single electron angular momentum rises to $l_{\max }=14$ when the cage is included in the final state, compared to $l_{\max }=8$ with the pure Coulombic final state.

\section{Conclusions}

In summary, we have computed double photoionization cross-sections from two-electron atoms endohedrally embedded in a model spherical fullerene. This simple system shows striking differences with the electronic emission from isolated atoms. The presence of the fullerene cage determines the structure of the cross-sections, breaking the initial state mirror collapse, and enhancing the role of higher angular momentum waves of the ejected electrons in the process. This effect is more remarkable for higher energies, although it can be seen for small ones, providing that the final state also includes the cage potential. The relation between the present results and confinement resonances found in this process deserves further explorations.

\section{Acknowledgments}

Authors would like to acknowledge the financial support of ANPCyT (PICT 0934/08) and CONICET (PIP 200901/552). G. Gasaneo would like to acknowledge the support of the Universidad Nacional del Sur (Argentina), PGI 24/F049. D. Mitnik acknowledges the support of the University of Buenos Aires (Argentina), UBACyT 239.

\section{References}

[1] H. W. Kroto, J. R. Heath, S. C. O’Brien, R. F. Curl, and R. E. Smalley, " $\mathrm{C}_{60}$ : buckminsterfullerene," Nature, vol. 318, no. 6042, pp. 162-163, 1985.

[2] D. S. Bethune, R. D. Johnson, J. R. Salem, M. S. De Vries, and C. S. Yannoni, "Atoms in carbon cages: the structure and properties of endohedral fullerenes," Nature, vol. 366, no. 6451, pp. 123-128, 1993.

[3] C. S. Yannoni, M. Hoinkis, M. S. De Vries et al., "Scandium clusters in fullerene cages," Science, vol. 256, no. 5060, pp. 1191-1192, 1992.

[4] O. V. Pupysheva, A. A. Farajian, and B. I. Yakobson, "Fullerene nanocage capacity for hydrogen storage," Nano Letters, vol. 8, no. 3, pp. 767-774, 2008.

[5] For a periodic table of endohedrally embedded atoms, http:// homepage.mac.com/jschrier/endofullerenes_table.html.

[6] M. J. Puska and R. M. Nieminen, "Photoabsorption of atoms inside $\mathrm{C}_{60}$," Physical Review A, vol. 47, no. 2, pp. 1181-1186, 1993.
[7] Y. B. Xu, M. Q. Tan, and U. Becker, "Oscillations in the photoionization cross section of $\mathrm{C}_{60}$," Physical Review Letters, vol. 76, no. 19, pp. 3538-3541, 1996.

[8] M. Y. Amusia, A. S. Baltenkov, and U. Becker, "Strong oscillations in the photoionization of 5 s electrons in Xe@ $\mathrm{C}_{60}$ endohedral atoms," Physical Review A, vol. 62, no. 1, Article ID 012701, 4 pages, 2000.

[9] M. Y. Amusia, A. S. Baltenkov, V. K. Dolmatov, S. T. Manson, and A. Z. Msezane, "Confinement resonances in photoelectron angular distributions from endohedral atoms," Physical Review A, vol. 70, no. 2, Article ID 023201, 5 pages, 2004.

[10] V. K. Dolmatov and S. T. Manson, "Correlation confinement resonances in photoionization of endohedral atoms: Xe@ $\mathrm{C}_{60}$ "' Journal of Physics B, vol. 41, no. 16, Article ID 165001, 2008.

[11] M. Y. Amusia, A. S. Baltenkov, and L. V. Chernysheva, "Photoionization of the subvalent subshells of noble gas endohedrals: interference of three resonances," Journal of Physics B, vol. 41, no. 16, Article ID 165201, 2008.

[12] P. N. Juranić, D. Lukić, K. Barger, and R. Wehlitz, "Experimental evidence for modulations in the relative doublephotoionization cross section of $\mathrm{C}_{60}$ from threshold up to 280 eV," Physical Review Letters, vol. 96, no. 2, Article ID 023001, 2006.

[13] O. Kidun, N. Fominykh, and J. Berakdar, "Multiple ionization of the fullerene by a single photon," Computational Materials Science, vol. 35, no. 3, pp. 354-358, 2006.

[14] M. Y. Amusia, E. Z. Liverts, and V. B. Mandelzweig, "Twoelectron photoionization of endohedral atoms," Physical Review A, vol. 74, no. 4, Article ID 042712, 2006.

[15] J. A. Ludlow, T. G. Lee, and M. S. Pindzola, “Time-dependent close-coupling calculations of the double photoionization of He@C 60 ," Physical Review A, vol. 81, no. 2, Article ID 023407, 2010.

[16] L. Avaldi and A. Huetz, "Photodouble ionization and the dynamics of electron pairs in the continuum," Journal of Physics B, vol. 38, no. 9, pp. S861-S891, 2005.

[17] D. M. Mitnik, J. Randazzo, and G. Gasaneo, "Endohedrally confined helium: study of mirror collapses," Physical Review A, vol. 78, no. 6, Article ID 062501, 2008.

[18] M. Neek-Amal, G. Tayebirad, and R. Asgari, "Ground-state properties of a confined simple atom by $\mathrm{C}_{60}$ fullerene," Journal of Physics B, vol. 40, no. 8, pp. 1509-1521, 2007.

[19] J. P. Connerade, K. V. Dolmatov, P. A. Lakshmi, and S. T. Manson, "Electron structure of endohedrally confined atoms: atomic hydrogen in an attractive shell," Journal of Physics B, vol. 32, no. 10, pp. L239-L245, 1999.

[20] J. P. Connerade, V. K. Dolmatov, and S. T. Manson, "Controlled strong non-dipole effects in photoionization of confined atoms," Journal of Physics B, vol. 33, no. 7, pp. L275L282, 2000.

[21] K. Govil and P. C. Deshmukh, "Quadrupole photoionization of endohedral Xe@C 60 ," Journal of Physics B, vol. 42, no. 17, Article ID 175003, 2009.

[22] J. A. Ludlow, J. Colgan, T. G. Lee, M. S. Pindzola, and F. Robicheaux, "Double photoionization of helium including quadrupole radiation effects," Journal of Physics B, vol. 42, no. 22, Article ID 225204, 2009.

[23] L. U. Ancarani, G. Gasaneo, F. D. Colavecchia, and C. Dal Cappello, "Interplay of initial and final states for $(e, 3 e)$ and $(\gamma, 2 e)$ processes on helium," Physical Review A, vol. 77 , no. 6 , Article ID 062712, 2008. 
[24] A. S. Kheifets and I. Bray, "Convergent calculations of double ionization of helium: from $(\gamma, 2 e)$ to $(\mathrm{e}, 3 e)$ processes," Physical Review A, vol. 69, no. 5, Article ID 050701, 2004.

[25] S. P. Lucey, J. Rasch, C. T. Whelan, and H. R. J. Walters, "Gauge discrepancies in calculations of $(\gamma, 2 e)$ on helium," Journal of Physics B, vol. 31, no. 6, pp. 1237-1258, 1998.

[26] T. Hahn, "Cuba-a library for multidimensional numerical integration," Computer Physics Communications, vol. 168, no. 2, pp. 78-95, 2005.

[27] S. Otranto and C. R. Garibotti, "Kinetic correlation in the final-state wave function in photo-double-ionization of He," Physical Review A, vol. 67, no. 6, Article ID 064701, 4 pages, 2003.

[28] J. Colgan and M. S. Pindzola, "Total and differential crosssection calculations for the double photoionization of the helium $1 \mathrm{~s} 2 s^{1,3} S$ states," Physical Review A, vol. 67, no. 1, Article ID 012711, 7 pages, 2003.

[29] P. Bolognesi, R. Flammini, A. Kheifets, I. Bray, and L. Avaldi, "Experimental observation of initial-state effects in photodouble-ionization of Ne 2s," Physical Review A, vol. 70, no. 6, Article ID 062715, 6 pages, 2004.

[30] P. Bolognesi, S. Otranto, C. R. Garibotti, R. Flammini, G. Alberti, and L. Avaldi, "Photo-double ionisation of the $\mathrm{Ne} 2 \mathrm{~s}$ studied in equal-energy-sharing condition at $20 \mathrm{eV}$ above threshold," Journal of Electron Spectroscopy and Related Phenomena, vol. 144-147, pp. 63-66, 2005.

[31] L. Malegat, P. Selles, and A. Huetz, "Double photoionization: I. A new parametrization of the triple differential cross section from first principles," Journal of Physics B, vol. 30, no. 2, pp. 251-261, 1997.

[32] L. Malegat, P. Selles, P. Lablanquie, J. Mazeau, and A. Huetz, "Double photoionization: II. Analysis of experimental triple differential cross sections in helium and neon," Journal of Physics B, vol. 30, no. 2, pp. 263-276, 1997. 

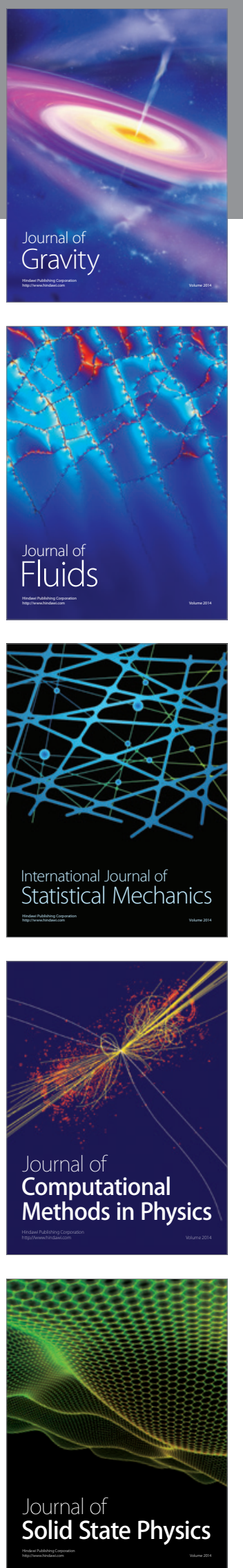

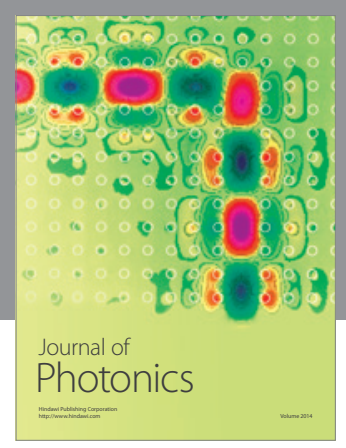

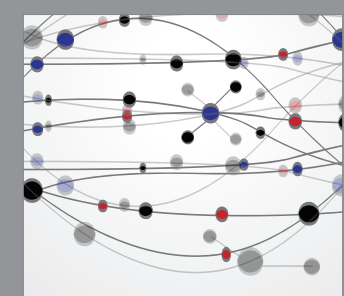

The Scientific World Journal
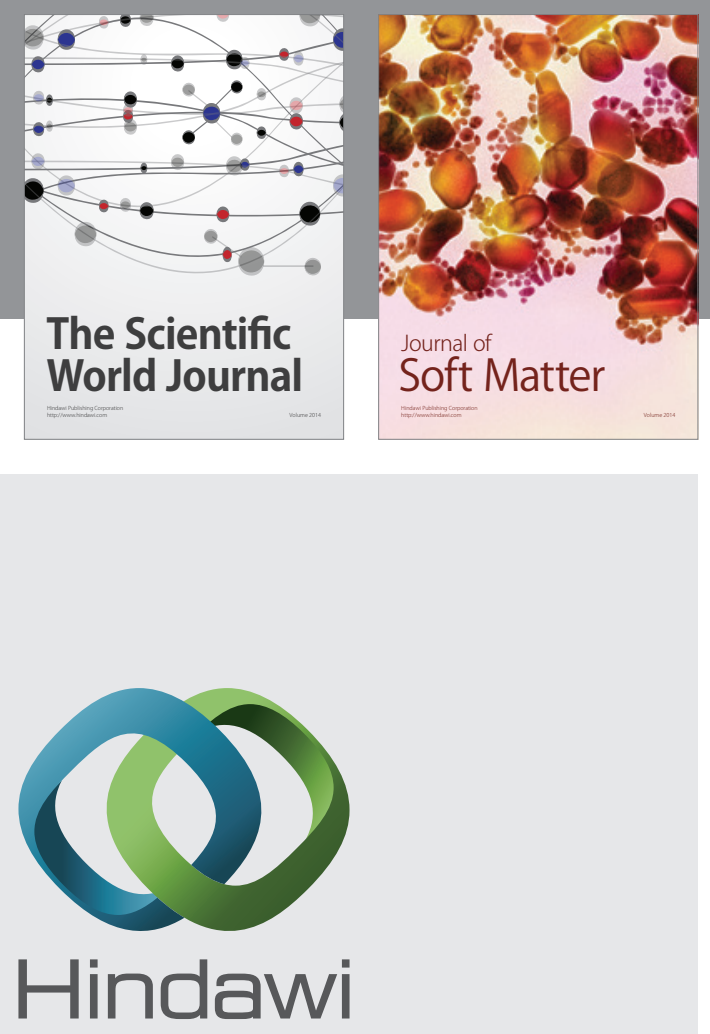

Submit your manuscripts at

http://www.hindawi.com
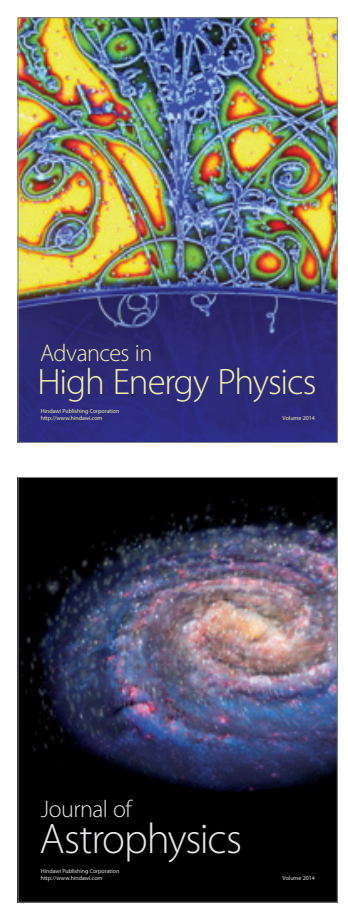
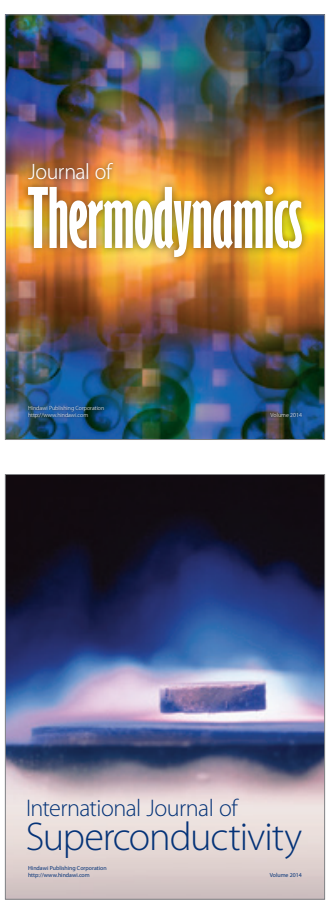
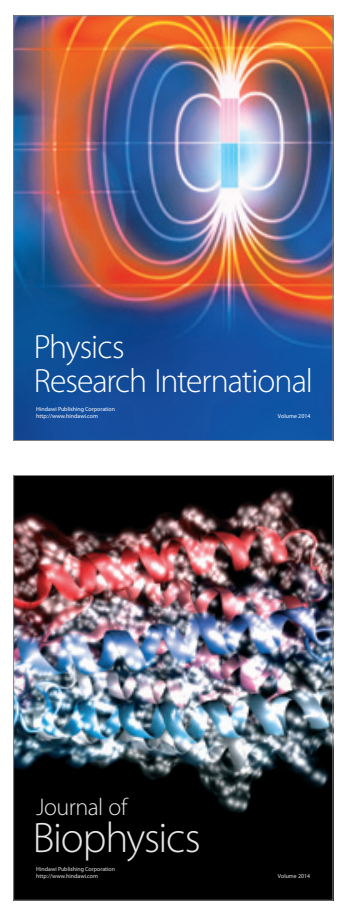
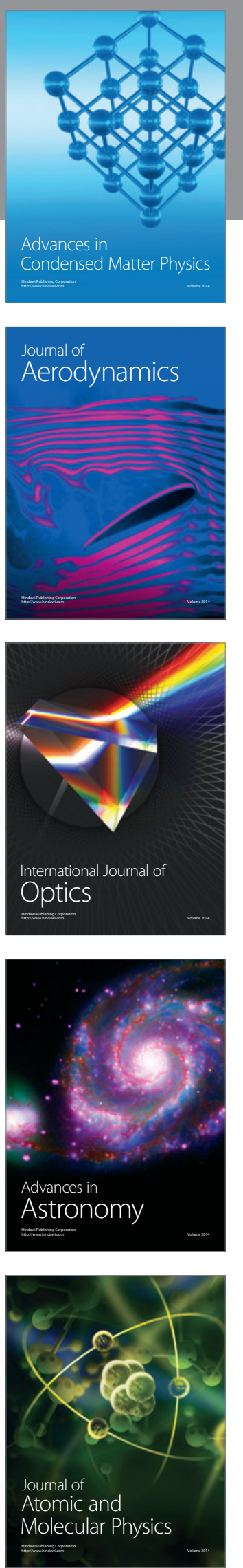